\title{
Etiological analysis of parastomal hernia by computed tomography examination
}

\author{
Yu Wei Pu, Xiao Dong Yang, Wei Gong, Chun Gen Xing \\ Department of General Surgery, The Second Affiliated Hospital, Soochow University, Suzhou, China
}

Videosurgery Miniinv 2019; 14 (3): 387-393

DOI: https://doi.org/10.5114/wiitm.2019.81409

\begin{abstract}
Introduction: Parastomal hernia is a common complication after stoma formation. The definitive risk factors for parastomal hernia development remain unclear.

Aim: This study evaluated the risk factors through computed tomography (CT) scan of patients with parastomal hernia. Material and methods: All patients who underwent an operation at our institution from January 2008 to February 2014 were included. We recorded patient-related and operation-related variables, and CT scans were checked. All the variables were analyzed with SPSS 19 to identify the risk factors for parastomal hernia formation.

Results: Of the 128 patients who underwent colostomy, 49 (38.3\%) developed a parastomal hernia during a median follow-up period of 20.1 months (range: 4-84 months). Hernia development was significantly associated with the thickness of subcutaneous fat in the abdominal wall, the location of the stoma, anteroposterior diameter and horizontal diameter of the body. The defect size of the abdominal wall is another risk factor. The larger the defect size of the abdominal wall, the larger is the parastomal stoma $(3.79 \pm 1.51 \mathrm{vs} .2 .13 \pm 0.74 \mathrm{~cm}$ horizontally and $4.90 \pm 2.25 \mathrm{vs}$. $2.94 \pm 0.73 \mathrm{~cm}$ vertically, $p<0.001)$. The hernia contents protrude into the hernial sac through the path of the inner side more than the outer side (77.6\% vs. $12.2 \%$ ).

Conclusions: Our findings in Chinese patients with parastomal hernia match those from Western countries: obesity, the location of the stoma, and the defect size of the abdominal wall are significant risk factors for parastomal hernia formation. The mesenteric region is a weak area, which is a site prone to parastomal hernia, and should be protected.
\end{abstract}

Key words: parastomal hernia, risk factors, computed tomography.

\section{Introduction}

Parastomal hernia is a common complication after sigmoid colostomy, and the incidence is different in different literatures, up to $52 \%$ [1-5]. The application of imaging technology makes the diagnosis of side parastomal hernia more certain, and the incidence can be as high as 78\% [6]. Patients usually have a high incidence within the first year of the operation, and it increases with the duration of follow-up [7-10]. Along with the rising incidence of colorectal cancer, this complication is more and more prominent. Because of hernia bulging, the ostomy bag was difficult to attach to the skin, and fecal overflow causes inflammation around the colostomy, leading to nursing difficulties $[11,12]$. A huge hernia can result in abdominal wall prolapse, in addition to the cosmetic effect, but more important is the effect on daily life, with lower quality of life. Serious parastomal hernia surgery is needed, but the complication rate of use of mesh patch repair surgery is high, and in severe cases can lead to death [13]. At present, there are two ways to prevent the occurrence of parastomal hernia. One is prophylactic

\section{Address for correspondence}

Chun Gen Xing MD, Department of General Surgery, The Second Affiliated Hospital, Soochow University, 215006 Suzhou, China, e-mail: sdzhmd@163.com 
mesh application during colostomy [14]. The other is to further study the mechanism, and change the traditional method of colostomy.

\section{Aim}

The diagnosis of parastomal hernia depends on physical examination. However, with the development of imaging, we can study more intuitively, then the imaging results showed that the actual parastomal hernia incidence is much higher than shown by physical examination $[3,6]$. The imaging data can provide more information, such as abdominal wall defect size, hernia contents and the prominent position of hernia contents, etc. Through the measurement function carried out by the software, the process of colostomy can be dynamically studied.

The analysis of the risk factors of hernia comes from the western countries, but whether it is suitable for Chinese patients is debatable. This is a retrospective study of 128 patients with sigmoid colostomy in our hospital from January 2008 to February 2014, using imaging data to study the characteristics of hernia, analyze the risk factors, infer the pathogenesis, and to provide a theoretical basis for prevention of its occurrence.

\section{Material and methods}

There were 128 patients, 80 males and $48 \mathrm{fe}$ males, age 28-88 years old (median: 62). There were 123 cases of rectal cancer (113 patients accepted

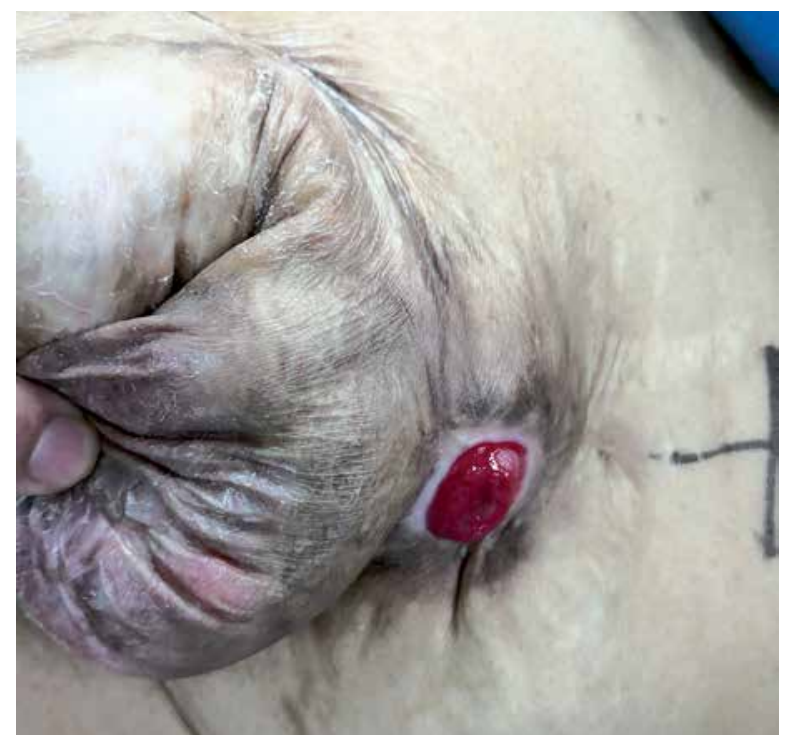

Photo 1. A 71-year-old woman with parastomal hernia had undergone Hartmann surgery
Miles surgery and 10 patients accepted Hartmann surgery), and 5 cases of rectal or sigmoid perforation. During the follow-up, the patients underwent physical examination, and 124 cases were examined by computed tomography (CT) and 4 cases were diagnosed by physical examination without CT (Photos 1, 2). The median follow-up period is 20.1 months (range: 4-84 months). The obvious bulging colostomy is the clinical diagnosis standard. Computed tomography diagnosis of abdominal CT was in accordance with Moreno-Matias [15]. After surgery, the patient was scheduled to hospitalization or outpatient follow-up. During the follow-up, the patients underwent routine examination, including physical examination, blood test, blood biochemical examination, and abdominal $\mathrm{X}$-ray examination. The information of patients, including gender, age, albumin, smoking status, presence of elevated intra-abdominal pressure situation (chronic cough, constipation, prostate hypertrophy), stoma separation, stoma infection, and stoma necrosis, was collected. Through the CT scan, we diagnosed the parastomal hernia, and recorded the position of the stoma, position of the hernia content bulging, type of parastomal hernia, measuring umbilical level subcutaneous fat thickness, thickness of right rectus muscle, umbilical horizontal anteroposterior diameter and the upper and lower horizontal size of the stoma.

\section{Statistical analysis}

We used SPSS 19 software for statistical analysis. The first step: the experimental group of parastomal hernia and the control group of non-parastomal her-

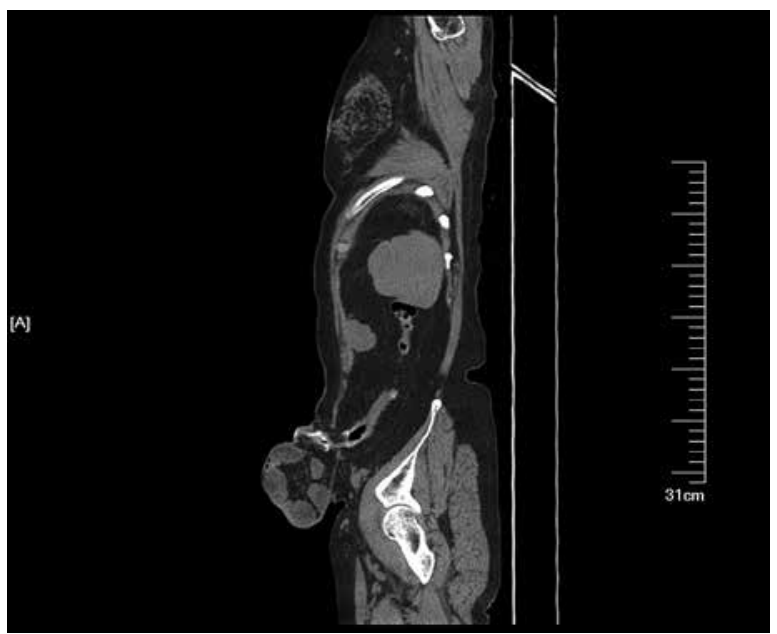

Photo 2. The CT scan of a 71-year-old woman with parastomal hernia 
nia make the Pearson $\chi^{2}$ and $T$ test separately to conduct risk factor analysis. According to the results of the first step, single factor analysis was performed for variables with significant statistical differences, into the Logistic regression model (backward) in search of the independent risk factors. The third, The relationship between the size of the hernia sac and the size of the abdominal wall defect was analyzed by paired-samples $T$ test (double side).

\section{Results}

The patient's clinical data are shown in Table I. This study included 128 patients, 80 males and 48 females; the median age was 62 (range: $28-88$ years old). There were $17(13.1 \%)$ cases of diabetes. There were 123 cases of rectal cancer (113 cases with the Mile's and Hartmann's 10 cases), 5 cases of rectal or sigmoid perforation. There were 14 (10.9\%) patients with perioperative colostomy separation, necrosis or infection. There were 32 (25\%) cases of laparoscopic surgery and 96 (75\%) cases of open operations. Follow-up time: 6-84 months (average: 20.1 months).

Of the 128 patients, 49 (38.3\%) cases had a parastomal hernia, and occurred at the earliest 4 months and 60 months at the latest after surgery. Statistical analysis showed that (Table II): the subcutaneous fat thickness ( $p<0.001$ ), waist circumference ( $p<0.001)$, stoma formation pass rectus abdominis $(p=0.02)$ and size of stoma are risk factors for parastomal hernia. The stoma size of patients with parastomal hernia is obviously greater than that of patients without parastomal hernia (3.79 \pm 1.51 vs. $2.13 \pm 0.74 \mathrm{~cm}$ horizontally and $4.90 \pm 2.25$ vs. 2.94 $\pm 0.73 \mathrm{~cm}$ vertically, $p<0.001$ ) (Table II).

In Table II, the items for which $p<0.05$ was obtained by the logistic regression model include level of umbilical horizontal fat thickness, umbilical horizontal anteroposterior diameter, the left and right umbilical horizontal diameter, the size of the abdominal wall defect (horizontal, upper and lower diameter) and the position of the stoma. The results showed that the thickness of subcutaneous fat, size of stoma and position of the stoma are independent risk factors (Table III).

With paired samples $T$ test analysis of the relationship between the abdominal wall defect size and the size of the hernial sac, the result showed that the greater the abdominal wall defect, the greater was the size of the hernial sac ( $p=0.001$, transverse
Table I. Clinical data

\begin{tabular}{|lc|}
\hline Parameter & Value \\
\hline Male & 80 \\
\hline Female & 48 \\
\hline Age [months] & 6-84 (mean: 20.1) \\
\hline \begin{tabular}{ll} 
Surgical method: \\
\hline Miles
\end{tabular} & 113 \\
\hline Hartmann's & 10 \\
\hline Sigmoid stoma & 52 \\
\hline Laparoscopic surgery & 96 \\
\hline Open operation & 14 \\
\hline $\begin{array}{l}\text { Colostomy separation, necrosis } \\
\text { or infection }\end{array}$ & 79 \\
\hline \begin{tabular}{l} 
Parastomal hernia: \\
\hline No
\end{tabular} & 49 \\
\hline Yes & 17 \\
\hline Diabetes & \\
\hline
\end{tabular}

dimension; $p=0.002$, vertical dimension). Hernial sac size is not associated with the location of the colostomy (Table IV).

The results showed that the majority of hernia contents bulging was located in the medial part of the stoma (including the upper medial and lower medial), which is $77.6 \%$ (38 patients). The rate of hernia contents bulging located at the lateral part of the stoma is $12.2 \%$ ( 6 patients) (including the upper lateral and lower lateral) (Table V).

\section{Discussion}

Parastomal hernia is a common long-term complication after enterostomy. There are many pathogenic factors, there is no consensus on the treatment method, and there is no recognized classification in the industry, so it is difficult to conduct a rigorous comparative study. The European Hernia Society (EHS) organized a special meeting of experts on parastomal hernia in Poland in April 2012. Based on the literature review of parastomal hernia, the current classification of parastomal hernia was evaluated and a new criterion for the classification of parastomal hernia was established [16]. Gil and 
Table II. Analysis of parastomal hernia risk factors (Pearson $\chi^{2}$ and $T$ test)

\begin{tabular}{|c|c|c|c|}
\hline Parameter & Parastomal hernia (+) & Parastomal hernia (-) & $P$-value \\
\hline Sex: & & & 0.542 \\
\hline Male & 29 & 51 & \\
\hline Female & 20 & 28 & \\
\hline Age & $62.96 \pm 10.90$ & $60.96 \pm 12.87$ & 0.368 \\
\hline Albumin & $37.68 \pm 13.06$ & $36.91 \pm 11.77$ & 0.730 \\
\hline Diabetes: & & & 0792 \\
\hline Yes & 7 & 10 & \\
\hline No & 42 & 69 & \\
\hline Smoking status: & & & 0.410 \\
\hline Yes & 13 & 16 & \\
\hline No & 36 & 63 & \\
\hline Laparoscopic surgery: & & & 0.852 \\
\hline Yes & 12 & 20 & \\
\hline No & 37 & 57 & \\
\hline Thickness of umbilical level subcutaneous [cm] & $2.06 \pm 0.71$ & $1.46 \pm 0.79$ & $<0.001$ \\
\hline Thickness of right rectus muscle $[\mathrm{cm}]$ & $0.99 \pm 0.27$ & $0.98 \pm 0.27$ & 0.834 \\
\hline Umbilical horizontal anteroposterior diameter [cm] & $21.01 \pm 2.60$ & $18.90 \pm 3.01$ & $<0.001$ \\
\hline Umbilical horizontal diameter [cm] & $31.37 \pm 2.62$ & $29.21 \pm 3.38$ & $<0.001$ \\
\hline Abdominal wall defect (horizontal) $[\mathrm{cm}]$ & $3.79 \pm 1.51$ & $2.13 \pm 0.74$ & $<0.001$ \\
\hline $\begin{array}{l}\text { Abdominal wall defect (the upper and lower } \\
\text { direction) }[\mathrm{cm}]\end{array}$ & $4.90 \pm 2.25$ & $2.94 \pm 0.73$ & $<0.001$ \\
\hline Stoma position: & & & 0.020 \\
\hline Pass rectus abdominis muscle (60) & 17 & 43 & \\
\hline Pass beside rectus abdominis muscle (66) & 32 & 34 & \\
\hline Colostomy separation, necrosis or infection: & & & 0.350 \\
\hline Yes & 7 & 7 & \\
\hline No & 42 & 72 & \\
\hline
\end{tabular}

Table III. Logistic stepwise regression model

\begin{tabular}{|lccc|}
\hline Parameter & $B$ value & Standard error & $P$-value \\
\hline Thickness of umbilical level subcutaneous & 1.007 & 0.404 & 0.013 \\
\hline Abdominal wall defect (horizontal) & 0.903 & 0.454 & 0.046 \\
\hline Abdominal wall defect (upper and lower direction) & 1.287 & 0.418 & 0.002 \\
\hline Stoma position & -1.430 & 0.833 & 0.086 \\
\hline Constant & -8.861 & 1.734 & $<0.001$ \\
\hline
\end{tabular}


Table IV. Relationship between size of abdominal wall defect and size of hernial sac

\begin{tabular}{|lccc|}
\hline & $\begin{array}{c}\text { Pass rectus } \\
\text { abdominis muscle }\end{array}$ & $\begin{array}{l}\text { Pass beside rectus } \\
\text { abdominis muscle }\end{array}$ & $P$-value \\
\hline Size of hernial sac (horizontal) $[\mathrm{cm}]$ & $8.17 \pm 2.99$ & $9.32 \pm 3.77$ & 0.278 \\
\hline $\begin{array}{l}\text { Size of hernial sac (the upper and lower } \\
\text { direction) }[\mathrm{cm}]\end{array}$ & $7.98 \pm 3.17$ & $9.31 \pm 3.44$ & 0.192 \\
\hline
\end{tabular}

Szczepkowski's classification includes concomitant midline incisional hernia [17]. In 2017, the European Hernia Society published guidelines on the prevention and treatment of parastomal hernia. This is the first international guideline for the treatment of parastomal hernia [18].

In recent years, the incidence of colorectal cancer has increased year by year. A number of patients need to undergo colostomy surgery, which will create the problem of psychological and physiological aspects. Parastomal hernia is the most common complication of colostomy, often leading to fecal leakage, skin inflammation, and decreased quality of life. A few patients will experience intestinal obstruction, small bowel incarceration, necrosis and perforation. It is important to distinguish between surgical and patient variables of the risk factors associated with the development of a parastomal hernia [19]. Pilgrim et al. reported 3 risk factors of parastomal hernial: (1) disease process factors: including obesity, diabetes, ulcerative colitis, other abdominal hernia, intra-abdominal pressure factors, chronic obstructive airway disease, postoperative infection, application of hormone and malignant disease. (2) Patient factors: including age, smoking and malnutrition. (3) Technical factors: including whether to perform an emergency operation and size of the stoma [20]. In the case of parastomal fistula repair, not only increased pain of the patient, but also the operation have certain complications. In 2013, Henriksen et al. reported 174 cases of parastomal hernia repair in which a synthetic material patch was placed extraperitoneally [21]. The complication rare of surgery was $13.2 \%$. Ninetten patients underwent a second operation and 6 cases of deep wound infection (3.45\%) were the most important causes. The mortality rate was $6.3 \%$ within 30 days. There are two existing countermeasures: one is the placement of mesh in the extraperitoneal area at the stoma formation, which is reported more in the European and American literature [14], and the other method is to change the position of the stoma or sigmoid colostomy.
Table V. Location of hernia contents bulging

\begin{tabular}{|lc|}
\hline Location of hernia contents bulging & $N$ \\
\hline Medial & 10 \\
\hline Lateral & 1 \\
\hline Upper & 1 \\
\hline Lower & 4 \\
\hline Upper medial & 17 \\
\hline Lower medial & 11 \\
\hline Upper lateral & 4 \\
\hline Lower lateral & 1 \\
\hline
\end{tabular}

In Europe and the United States, the operation of placement of the mesh extraperitoneally to prevent parastomal hernia in the literature was reported. Zhu et al. and Cornille et al. conducted a meta-analysis to evaluate the value of this operation $[14,22]$. The results showed that the operation decreased the rate of parastomal hernia obviously and did not increase colostomy complications. But the economics of the operation and postoperative quality of life of patients are reported rarely. The sample size was not sufficiently large, and this might have resulted in bias, so the value of the operation is still controversial.

Computed tomography examination can be a specific diagnosis of parastomal hernia and the results show that the detection rate of $\mathrm{CT}$ is higher than that of simple physical examination, up to 4 times[23-25]. Computed tomography imaging can not only confirm whether there is a hernia, but also provide more information, including the size of the hernia and the position of hernia contents prominence, and provides a method of measurement. Studies indicate the risk factors of parastomal hernia: female, high body mass index, low albumin, laparoscopic surgery and peritoneal colostomy, and the location of colostomy [26, 27]. The data analysis of the high body mass index and colostomy position is consistent with our study. 
According to the results of this study, the thickness of abdominal subcutaneous fat, umbilical anteroposterior diameter and horizontal diameter of patients with parastomal hernial are obviously greater than those of patients without parastomal hernia. These indicators point to the extent of the patient's obesity and obese patients are more likely to have parastomal hernia. Obese patients have more fat tissue around their colon and larger bowel diameters. Therefore, a larger abdominal wall defect is required for the stoma. The adipose tissue adjacent to the colon is trimmed before colostomy, as much as possible to remove mesocolic adipose tissue.

This study showed that the size of the abdominal wall defect is related to the formation of hernia. We measured the defect size of the abdominal wall defect horizontally and vertically; the results showed that patients with hernia have larger dimensions than patients with no hernia in both directions (respectively $3.79 \pm 1.51$ vs. $2.13 \pm 0.74 \mathrm{~cm}, 4.90 \pm 2.25$ vs. $2.94 \pm 0.73 \mathrm{~cm}, p<0.001)$. Based on the results, we suggested that colostomy abdominal wall defect diameter should be controlled below $3 \mathrm{~cm}$. Colostomy location is another recognized risk factor. This study has the same result that the stoma pass rectus abdominis muscle, the incidence of the hernia is low. How to protect the mesangial area is an important issue. Through the way of extraperitoneal colostomy, in association with dragging out the process of rotating insufflate $180^{\circ}$, mesangial is located in the lateral, rear insufflate protection. This may reduce the parastomal hernia incidence.

This study showed that the majority of hernia contents bulging was located medially of the stoma, which is $77.6 \%$ (38 patients), and the rate of hernia contents bulging located laterally of the stoma is $12.2 \%$ (6 patients). Colostomy surgery requires protection of the blood supply of the colon, so the colostomy mesangial area is generally located in the medial colostomy area and this coincides with the prominent position of the hernia contents. The result indicated that the colostomy mesangial area is the weak area. Thus how to protect the colon mesangial area is an important issue. We hypothesized that through the way of extraperitoneal colostomy, rotary the bowel $180^{\circ}$ in the proceed of dragging out, then the colon mesangial located in the lateral and the posterior has bowl protection, and this may reduce the parastomal hernia incidence.

\section{Conclusions}

The risk factors of parastomal hernia after colostomy were obesity, colostomy position, and large abdominal wall defect. The mesangial area of the colostomy is the weak area where the majority of hernia contents bulging is located. The appropriate dressing colon surrounding adipose tissue, controlling the size of the abdominal wall defect, selecting the rectus colostomy and protecting the mesangial area will help reduce parastomal hernia.

\section{Conflict of interest}

The authors declare no conflict of interest.

\section{References}

1. Pearl RK. Parastomal hernias. World J Surg 1989; 13: 569-72.

2. Londono-Schimmer EE, Leong AP, Phillips RK. Life table analysis of stomal complications following colostomy. Dis Colon Rectum 1994; 37: 916-20.

3. Cheung MT, Chia NH, Chiu WY. Surgical treatment of parastomal hernia complicating sigmoid colostomies. Dis Colon Rectum 2001; 44: 266-70.

4. Makela JT, Turku PH, Laitinen ST. Analysis of late stomal complication following ostomy surgery. Ann Chir Gynaecol 1997; 86: 305-10.

5. Israelsson LA. Parastomal hernias. The Surgical Clinics of North America. Surg Clin North Am 2008; 88: 113-25.

6. Cingi A, Cakir T, Sever A, et al. Enterostomy site hernias: a clinical and computerized tomographic evaluation. Dis Colon Rectum 2006; 49: 1559-63.

7. Janes A, Cengiz Y, Israelsson LA. Preventing parastomal hernia with a prosthetic mesh. Arch Surg 2004; 139: 1356-8.

8. Janes A, Cengiz $Y$, Israelsson LA. Preventing parastomal hernia with a prosthetic mesh: a 5-year follow-up of a randomized study. World J Surg 2009; 33: 118-23.

9. Janes A, Weisby L, Israelsson LA. Parastomal hernia: clinical and radiological definitions. Hernia 2011; 15: 189-92.

10. Carne PW, Robertson GM, Frizelle FA. Parastomal hernia. $\mathrm{Br}$ J Surg 2003; 90: 784-93.

11. Hardt J, Meerpohl JJ, Metzendorf MI, et al. Lateral pararectal versus transrectal stoma placement for prevention of parastomal herniation. Cochrane Database Syst Rev 2013; 11: CD009487.

12. Al Shakarchi J, Williams JG. Systematic review of open techniques for parastomal hernia repair. Tech Coloproctol 2014; 18 : 427-32.

13. Goligher JC, Lloyd-davies OV, Robertson CT. Small gut obstructions following combined excision of the rectum with special reference to strangulation round the colostomy. Br J Surg 1951; 38: 467-73.

14. Zhu J, Pu Y, Yang X, et al. Prophylactic mesh application during colostomy to prevent parastomal hernia: a meta-analysis. Gastroenterol Res Pract 2016; 2016: 1694265.

15. Moreno-Matias J, Serra-Aracil X, Darnell-Martin, et al. The prevalence of parastomal hernia after formation of an end colosto- 
my. A new clinic-radiological classification. Colorectal Dis 2009; 11: $173-7$.

16. Śmietański M, Szczepkowski M, Alexandre JA, et al. European Hernia Society classification of parastomal hernias. Hernia 2014; 18: 1-6.

17. Gil G, Szczepkowski MS. A new classification of parastomal hernias from the experience at Bielański Hospital in Warsaw. Pol J Surg 2011; 83: 430-7.

18. Antonious SA, Agresta F, Garcia AJM, et al. European Hernia Society Guidelines on prevention and treatment of parastomal hernias. Hernia 2018; 22: 183-8.

19. Styliński R, Alzubedi A, Rudzki S. Parastomal hernia - current knowledge and treatment. Videosurgery Miniinv 2018; 13: 1-8.

20. Pilgrim CH, Mclntyre R, Bailey M. Prospective audit of parastomal hernia: prevalence and associated comorbidities. Dis Colon Rectum 2010; 53: 71-6.

21. Henriksen NA, Helgstrand F, Vogt KC, et al. Risk factors for incisional hernia repair after aortic reconstructive surgery in a nationwide study. I Vasc Surg 2013; 57: 1524-30.

22. Cornille JB, Pathak S, Daniels IR, et al. Prophylactic mesh use during primary stoma formation to prevent parastomal hernia. Ann R Coll Surg Engl 2017; 99: 2-11.

23. Serra-Aracil X, Bombardo-Junca J, Moreno-Matias J, et al. Randomized, controlled, prospective trial of the use of a mesh to prevent parastomal hernia. Ann Surg 2009; 249: 583-7.

24. Lambrecht JR, Larsen SG, Reiertsen O, et al. Prophylactic mesh at end-colostomy construction reduces parastomal hernia rate: a randomized trial. Colorectal Dis 2015; 17: 191-7.

25. Vierimaa M, Klintrup K, Biancari F, et al. Prospective, random ized study on the use of a prosthetic mesh for prevention of parastomal hernia of permanent colostomy. Dis Colon Rectum 2015; 58: 943-9.

26. Raet JD, Delvaux G, Haentjens P, et al. Waist circumference is an independent risk factor for the development of parastomal hernia after permanent colostomy. Dis Colon Rectum 2008; 51: 1806-9.

27. Funahashi K, Suzuki T, Nagashima Y, et al. Risk factors for parastomal hernia in Japanese patients with permanent colostomy. Surg Today 2014; 44: 1465-9.

Received: 22.09.2018, accepted: 17.12.2018. 